\title{
Modelling Set-up Times Overlapping Two Periods in the Proportional Lot-Sizing Problem with Identical Parallel Machines
}

\author{
Waldemar Kaczmarczyk*
}

\begin{abstract}
This paper presents a new mixed integer programming model for the Proportional Lot-Sizing Problem (PLSP) with identical parallel machines and set-up times overlapping two periods. The proposed model assumes constant period length and explicitly calculates the distribution of set-up operations among periods. The presented results of computational experiments with standard MIP methods prove that the untying set-ups from period borders enables the reduction of the total costs in optimal solutions.
\end{abstract}

Keywords: production planning, lot-sizing and scheduling, mixed integer programming

Mathematics Subject Classification: 90B30; 90C11

Revised: December 3, 2013

\section{INTRODUCTION}

This paper addresses mixed-integer programming (MIP) models for the Proportional Lot-sizing and Scheduling Problem (PLSP) proposed by Haase (1994) (see also Drexl and Haase, 1995). Lot-sizing and scheduling problems describe the scheduling of production lots for several products with deterministic, dynamic demand, on machines with limited capacity. The PLSP assumes, that at most one set-up operation may be executed on a single machine within a single period.

In this paper a new model formulation is presented for the PLSP with identical parallel machines (Kaczmarczyk, 2011), which allows set-up operations to overlap two periods. Increasing the capabilities of computers and MIP solvers increases the possibility of applying standard MIP methods to solve real planning problems. It is however necessary to describe them using appropriate model formulations (Belvaux and Wolsey, 2001). The aim of this paper is to find formulations of the model which could be solved using standard MIP software.

Set-up times overlapping multiple periods are important for several reasons. Increases in the computational capability of enterprise information systems allow for

* AGH University of Science and Technology, Faculty of Management, Department of Operations Research and Information Technology, Krakow, Poland. e-mail: waldek@agh.edu.pl 
more detailed planning, among other things planning based on a more detailed calendar. Thus, in the future, set-up times may become "longer" in relation to the time buckets used in planning (Suerie, 2006). The assumption, that a set-up operation can not overlap multiple periods, has two significant disadvantages. First, the time buckets have to be longer than the largest set-up time, what may prevent detailed planning. Long periods make it difficult to plan small lots, as they do not preserve high utilization of period capacity. Second, if the execution of a set-up operations is limited by period boundaries then some good solutions may be cut off from the solution space.

There are only a few lot-sizing and scheduling models considering long set-up times, all of them for the case with a single machine. In Cattrysse et al. (1993) and Blocher et al. (1999) the Discrete Lot-sizing and Scheduling Problem (DLSP) has been adapted for the set-up times overlapping multiple periods. The DLSP restricts lot-sizes and set-up times to multiples of a period's length. Haase (1994) proposed the first PLSP model formulation which allows that set-up operations overlap multiple periods. His model uses variables accumulating time assigned in consecutive periods to set-up operations. Suerie (2006) proved that this model is not correct and proposed two new models refining the idea from Haase (1994). Finally, Kaczmarczyk (2011) presented another PLSP formulation assuming constant period length.

The PLSP with parallel machines can be modelled using separable sets of binary variables and constraints for all machines (Kimms and Drexl, 1998). Using binary variables to describe identical parallel machines may lead to many solutions which differ only in the numbering of machines. These alternative solutions significantly increase the computational effort of the branch-and-cut algorithm. At a practical level, identical solutions are examined many times. Therefore, Kaczmarczyk (2011) proposed a new model, denoted PLSP/F, which aggregates the machines and uses integer variables. This model uses sequence-dependent change-over variables to describe a flow of machines set-up state between products. General surveys on lot-sizing and scheduling models can be found in Drexl and Kimms (1997) and Wolsey (2002).

This paper presents a new formulation of the PLSP/F model which allows for set-up operations to be split among two consecutive periods in the case with constant period length. The next section presents the basic model with identical parallel machines. Section 3 presents a new model formulation. Section 4 provides computational results. Finally, Section 5 gives a summary.

\section{MODEL WITH IDENTICAL PARALLEL MACHINES}

The Proportional Lot-sizing and Scheduling Problem (PLSP) (Haase, 1994) is a small bucket model, i.e. it allows at most one set-up operation within a single period. It is the most flexible among small bucket models, as it allows for the processing of two products within a single period, one before and another after the set-up operation.

In Table 1 parameters and variables are presented common to both models presented in this paper. 
Table 1. Common parameters and variables

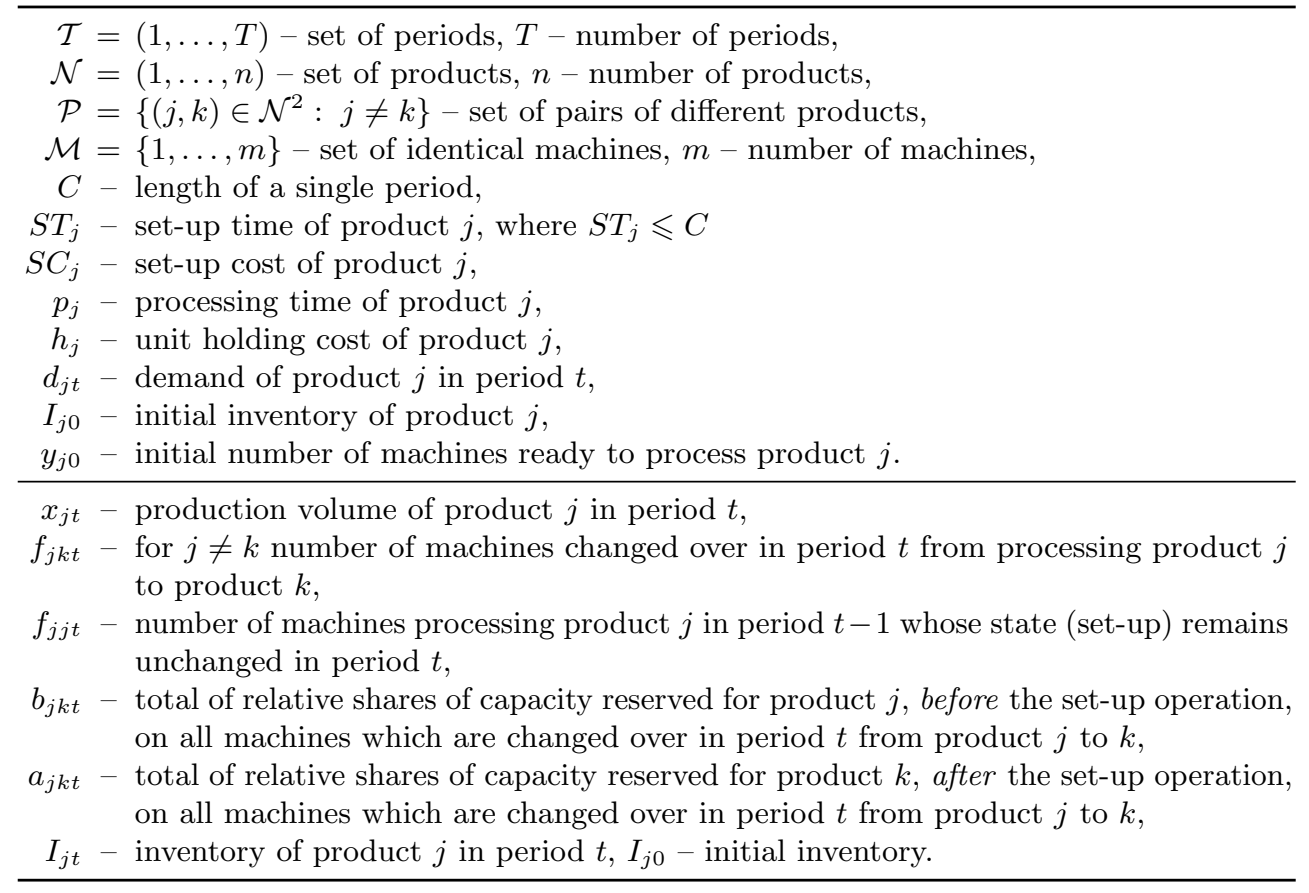

All variables with period index $t \leqslant 0$ are assumed to be to equal 0 . There are two exceptions from this rule, the initial inventories $I_{j 0}$ and the initial state of the machines $y_{j 0}$. It is assumed that $\sum_{j \in \mathcal{N}} y_{j 0}=m$, i.e. the initial set-up of machines is feasible. The PLSP/F model (Kaczmarczyk, 2011) may be stated as follows:

$$
\begin{aligned}
& \min \sum_{t \in \mathcal{T}} \sum_{j \in \mathcal{N}}\left(h_{j t} I_{j t}+S C_{j} \sum_{k \in \mathcal{N}: k \neq j} f_{k j t}\right) \\
& I_{j, t-1}+x_{j t}=d_{j t}+I_{j t}, \quad t \in \mathcal{T}, j \in \mathcal{N} \\
& f_{j j t}+\sum_{k \in \mathcal{N}: k \neq j}\left(b_{j k t}+a_{k j t}\right) \geqslant\left(p_{j} / C\right) x_{j t}, \quad t \in \mathcal{T}, j \in \mathcal{N} \\
& f_{j k t}\left(1-S T_{k} / C\right)=b_{j k t}+a_{j k t}, \quad t \in \mathcal{T},(j, k) \in \mathcal{P} \\
& f_{j j 0}=y_{j 0}, \quad j \in \mathcal{N} \\
& f_{j k 0}=0, \quad(j, k) \in \mathcal{P} \\
& \sum_{k \in \mathcal{N}} f_{k j, t-1}=\sum_{k \in \mathcal{N}} f_{j k t}, \quad t \in \mathcal{T}, j \in \mathcal{N} \\
& \sum_{(j, k) \in \mathcal{N}^{2}} f_{j k T}=m, \quad t \in \mathcal{T}, j \in \mathcal{N} \\
& x_{j t}, I_{j t} \geqslant 0, \quad t \in \mathcal{T}, j \in \mathcal{N}
\end{aligned}
$$




$$
\begin{aligned}
b_{j k t}, a_{j k t} & \in[0, m], & & t \in \mathcal{T},(j, k) \\
f_{j k t}, & \in\{0, \ldots, m\}, & t \in \mathcal{P},(j, k) & \in \mathcal{N}^{2}
\end{aligned}
$$

The objective (1a) is to minimize the set-up and inventory holding costs. The constraints (1b) preserve a feasible balance of inventory, production and demand. The constraints (1c) limit the production volume: variable $f_{j j t}$ describe the number of machines which continue to process product $j$ and expression $\sum_{k \in \mathcal{N}: k \neq j}\left(b_{j k t}+a_{k j t}\right)$ determine the share of capacity reserved for product $j$ on all machines which are changed over in this period. The constraints (1d) ensure that the sum of $b_{j k t}$ and $a_{j k t}$ variables does not exceed the capacity of all machines changed over from product $j$ to $k$ after the subtraction of set-up times.

Variables $f_{j k t}$ are the only integer variables in this model. Equations (1e-h) ensure that $f_{j k t}$ take feasible values, i.e. they preserve the flow of machine set-up units from product $j$ to product $k$ over time (Karmarkar and Schrage, 1985; Wolsey, 1989). Here, the variable $f_{j k t}$ is the flow from node $(j, t-1)$ to $(k, t)$, indicating that a number of machines have changed over from the set-up of item $j$ in period $t-1$ to the set-up of item $k$ in period $t$.

\section{MODEL WITH SET-UPS OVERLAPPING TWO PERIODS}

In this paper a new PLSP model formulation for identical parallel machines and set-up operations overlapping two periods is presented, denoted further as PLSP/F-LST. In Table 2 additional variables are presented, specific to the new model.

Table 2. Additional variables

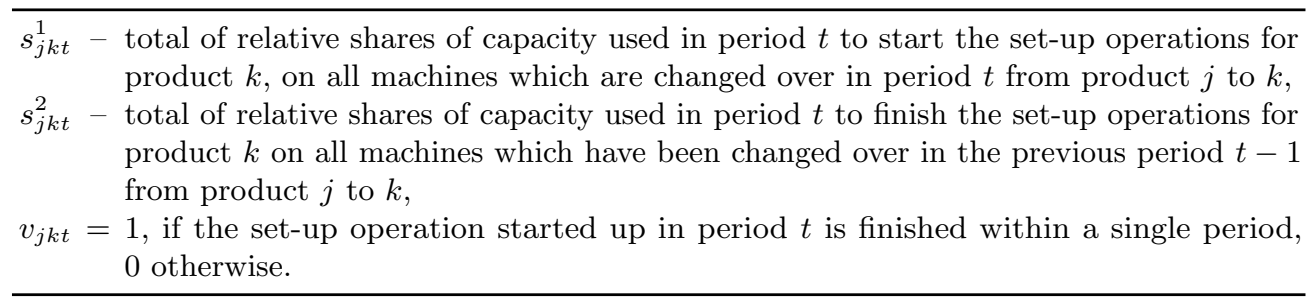

The PLSP/F-LST model for identical parallel machines and set-up operations overlapping two periods may be stated as follows:

$$
\begin{gathered}
\min \sum_{t \in \mathcal{T}} \sum_{j \in \mathcal{N}}\left(h_{j t} I_{j t}+S C_{j} \sum_{k \in \mathcal{N}: k \neq j} f_{k j t}\right) \\
f_{j j t}+\sum_{k \in \mathcal{N}: k \neq j}\left(b_{j k t}+a_{k j t}-s_{k j t}^{1}-s_{k j t}^{2}\right) \geqslant \\
\geqslant\left(p_{j} / C\right) x_{j t}, \quad t \in \mathcal{T}, j \in \mathcal{N} \\
(t \in \mathcal{T}, j \in \mathcal{N}
\end{gathered}
$$




$$
\begin{aligned}
& b_{j k t}+a_{j k t}=f_{j k t}, \quad t \in \mathcal{T},(j, k) \in \mathcal{P} \\
& f_{j j 0}=y_{j 0}, \quad j \in \mathcal{N} \\
& f_{j k 0}=0, \quad(j, k) \in \mathcal{P} \\
& \sum_{k \in \mathcal{N}} f_{k j, t-1}=\sum_{k \in \mathcal{N}} f_{j k t}, \quad t \in \mathcal{T}, j \in \mathcal{N} \\
& \sum_{(j, k) \in \mathcal{N}^{2}} f_{j k T}=m, \quad t \in \mathcal{T}, j \in \mathcal{N} \\
& x_{j t}, I_{j t} \geqslant 0, \quad t \in \mathcal{T}, j \in \mathcal{N} \\
& b_{j k t}, a_{j k t} \in[0, m], \quad t \in \mathcal{T},(j, k) \in \mathcal{P} \\
& f_{j k t}, \in\{0, \ldots, m\}, \quad t \in \mathcal{T},(j, k) \in \mathcal{N}^{2} \\
& a_{j k t}-\left(S T_{k} / C\right) f_{j k t} \leqslant m v_{j k t}, \quad t \in \mathcal{T},(j, k) \in \mathcal{P} \\
& f_{j k T} \leqslant m v_{j k T}, \quad(j, k) \in \mathcal{P} \\
& \left(S T_{k} / C\right) f_{j k t}-m\left(1-v_{j k t}\right) \leqslant s_{j k t}^{1}, \quad t \in \mathcal{T},(j, k) \in \mathcal{P} \\
& a_{j k t}-m v_{j k t} \leqslant s_{j k t}^{1}, \quad t \in \mathcal{T},(j, k) \in \mathcal{P} \\
& \left(S T_{k} / C\right) f_{j k t}-a_{j k t} \leqslant s_{j k t}^{2}, \quad t \in \mathcal{T},(j, k) \in \mathcal{P} \\
& f_{j k t} \geqslant v_{j k t}, \quad t \in \mathcal{T},(j, k) \in \mathcal{P} \\
& \left(S T_{k} / C\right) f_{j k t}=s_{j k t}^{1}+s_{j k, t+1}^{2}, \quad t \in \mathcal{T},(j, k) \in \mathcal{P} \\
& s_{j k t}^{1}, s_{j k t}^{2} \in[0, m], \quad t \in \mathcal{T},(j, k) \in \mathcal{P} \\
& v_{j k t} \in\{0,1\}, \quad t \in \mathcal{T},(j, k) \in \mathcal{N}^{2}
\end{aligned}
$$

The new model is an extension of the PLSP/F model (1). In the PLSP/F-LST the objective function (1a) and constraints (2e-k) remain unchanged. The PLSP/F constraints (1c) and (1d) are replaced by constraints (2c) and (2d). In model (1), the variable $a_{j k t}$ does not contain the set-up time. In constraint (2c) of the new model, the time devoted to the execution of set-up operations $s_{j k t}^{1}$ is subtracted from the capacity reserved for production, therefore does not have to be excluded in ( $2 \mathrm{~d})$ from variable $a_{j k t}$.

To ensure proper values of new variables some new constraints are necessary. Constraint (2l) determines if the set-up operation can be finished within a single period or if it has to be continued in the next period. According to inequality $(2 \mathrm{~m})$, set-up operations started in the last period must be finished within a single period. The constraints $(2 \mathrm{n}-\mathrm{o})$ ensure the proper values of the setup execution time in the period, in which it begins, $(2 \mathrm{n})$ when the whole operation is executed within a single period and (2o) when it has to overlap two periods. The constraints $(2 \mathrm{p})$ determine the time necessary to finish the set-up operations overlapping the two periods.

Two valid inequalities make the model easier to solve with standard MIP methods. Constraint $(2 \mathrm{q})$ sets value of variable $v_{j k t}$ to zero if there is no changeover. Constraint (2r) forces the sum of set-up execution variables $s_{j k t}^{1}$ and $s_{j k, t+1}^{1}$ to be equal to the set-up length. 


\section{EXPERIMENTS AND DATA SETS}

To verify the complexity of the new model 15 instances of the problem have been randomly generated. Every instance has 5 products and 30 periods. The design of the instances is based on experience with lot-sizing and scheduling in the electronics industry. All the following parameters were randomly generated as integer numbers from a uniform distribution.

At first, demand $d_{j t}$ was drawn from the range $[1,100]$ and next with probability 0.4 set equal to 0 . To preserve the existence of a feasible solution, the demand during the first five periods for all products was set equal to 0 . The constant capacity $C$ of a single machine has been chosen in a way that preserves the utilisation of all machines in the range $[80 \%, 90 \%]$. In the calculation of utilisation, 3 start-up times per product were added to the total workload. The actual number of start-ups per machine in the calculated solutions was in the range [1.4,6.7]. The parameters: processing times $p_{j}$, unit holding costs $h_{j}$, set-up times $S T_{j}$ and costs $S C_{j}$, were randomly chosen from ranges $[1,5],[1,5],[0.2 C, 0.8 C],\left[0.1 H_{j}, 1.5 H_{j}\right]$ respectively, where $C$ is the period length and $H_{j}=h_{j} / C / p_{j}$ is the holding cost of the maximal single period production.

It turned out that it is not possible to solve the PLSP/F-LST model from scratch in a reasonable time with standard MIP procedures. Therefore, a simple warm start heuristic was applied. Firstly, the PLSP/F model with a 10 minute time limit was solved and then values of the set-up variables from its solution were passed to the PLSP/F-LST model. The time limit was again set to 10 minutes.

Table 3. Results

\begin{tabular}{llrrr}
\hline & & \multicolumn{3}{c}{ Number of machines } \\
\cline { 3 - 5 } & Model & 5 & 10 & 15 \\
\hline $\begin{array}{l}\text { Relative decrement of total cost [\%] } \\
\text { in PLSP/F-LST relative to PLSP/F }\end{array}$ & & 3.4 & 1.3 & 0.8 \\
\hline MIP gap [\%] & PLSP/F & 0.0 & 0.0 & 0.0 \\
& PLSP/F-LST & 4.0 & 1.6 & 1.2 \\
\hline Computation time [s] & PLSP/F & 33.6 & 21.8 & 33.9 \\
& PLSP/F-LST & 573.2 & 515.9 & 493.2 \\
\hline
\end{tabular}

The results of the experiments are presented in Table 3. The largest average cost reduction $3.4 \%$ has been achieved for 5 machines and decreases with the increment of machine number. The PLSP/F model was always solved to optimality within the time limit, as well as most instances of the PLSP/F-LST.

In Figure 1 the number of set-up operations per machine in final solutions and the relative decrement of total costs in the PLSP/F-LST model for various demand instances are presented. Instances are sorted in order of increasing set-up numbers. Cost decrement is closely related to the number of set-ups. The PLSP/F-LST model allows to decrease total costs in average by $0.2 \%$ for every changeover. 


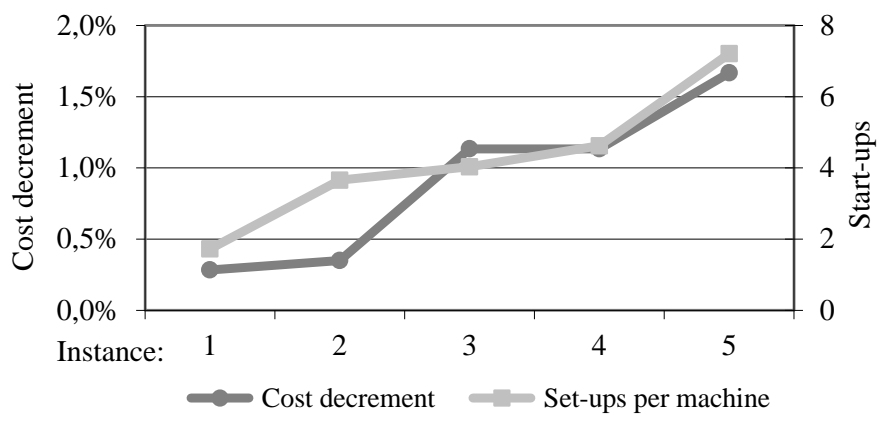

Fig. 1. Cost decrement for various demand instances

\section{SUMMARY}

In this paper a new model formulation for Proportional Lot-sizing and Scheduling Problem (PLSP) with identical parallel machines is presented which allows that the set-up times split among two periods. The results of the computational experiments prove that the presented model makes it possible to find better schedules than with a model with set-up operations executed within a single period, especially for problems with many changeovers in optimal solutions.

\section{REFERENCES}

Belvaux G., Wolsey L.A., 2001. Modelling Practical Lot-Sizing Problems as Mixed-Integer Programs, Management Science, 47(7), pp. 993-1007.

Blocher J.D., Chand S., Sengupta K., 1999. The changeover scheduling problem with time and cost considerations: Analytical results and a forward algorithm, Operations Research, 47, pp. 559-569.

Cattrysse D., Salomon M., Kuik R., van Wassenhove L., 1993. A dual ascent and column generation heuristic for the discrete lotsizing and scheduling problem with setup times, Management Science, 39, pp. 477-486.

Drexl A., Haase K., 1995. Proportional lotsizing and scheduling, International Journal of Production Economics, 40, pp. 73-87.

Drexl A., Kimms A., 1997. Lot sizing and scheduling - survey and extensions, European Journal Operational Research, 99, pp. 221-235.

Haase K., 1994. Lotsizing and scheduling for production planning. No. 408 Lecture Notes in Economics and Mathematical Systems, Springer-Verlag.

Kaczmarczyk W., 2009. Modelling Multi-Period Set-up Times in the Proportional Lot-Sizing Problem, Decision Making in Manufacturing and Services, 3, pp. 15-35.

Kaczmarczyk W., 2011. Proportional lot-sizing and scheduling problem with identical parallel machines, International Journal of Production Research, 49(9), pp. 2605-2623.

Karmarkar U.S., Schrage L., 1985. The Deterministic Dynamic Product Cycling Problem. Operations Research, 33(2), pp. 326-345.

Kimms A., Drexl A., 1998. Proportional lotsizing and scheduling: some extensions, Networks, 32(2), pp. 85-101. 
Suerie C., 2006. Modeling of period overlapping setup times, European Journal of Operational Research, 174(2), pp 874-886.

Wolsey L.A., 1989. Uncapacitated Lot-Sizing Problems With Start-Up Costs, Operations Research, 37(5), pp. 741-747.

Wolsey L.A., 2002. Solving Multi-Item Lot-Sizing Problems with an MIP Solver Using Classification and Reformulation, Management Science, 48(12), pp. 1587-1602. 